\title{
SINTOMAS URINÁRIOS E INTESTINAIS EM CRIANÇAS DA REDE PÚBLICA DE ENSINO FUNDAMENTAL
}

\author{
Camilla Pinheiro Cristaldi da Silva ${ }^{1}$ (D), Juliane Nascimento Ribas Miranda (D), \\ Drielle Fernanda Arruda2,* (D), Gisele Martins 3 (D), Gisela Maria Assis 4 (D)
}

\begin{abstract}
RESUMO
Objetivo: levantar a ocorrência de sintomas urinários e intestinais em crianças da rede pública de ensino fundamental da capital paranaense. Método: estudo quantitativo, exploratório-descritivo e de corte transversal. Amostra de pais/responsáveis que preencheram os seguintes instrumentos: Dysfunctional Voiding Scoring System; critérios de Roma IV; escala de Bristol. Análise realizada pelo IBM SPSS Statistics v.20.0. Os dados foram coletados no período de agosto a outubro de 2019. Os critérios de inclusão eram a criança estar matriculada e frequentando regularmente o ensino fundamental e a entrega do questionário preenchido ou parcialmente preenchido. Resultados: Foram entregues 458 questionários. Dos totalmente preenchidos, $51,9 \%$ era de meninas, e a de idade foi de 7,7 anos; $83 \%$ das crianças apresentaram algum sintoma urinário e/ou intestinal de baixa gravidade. Sem diferença significativa de sintomas entre sexos, e com acréscimo significativo de gravidade em crianças com 7 anos ou menos. Sintomas mais prevalentes: frequência miccional reduzida, frequência evacuatória reduzida, esforço evacuatório, urgência miccional e manobras de contenção; 39,6\% das crianças apresentavam Constipação Intestinal Funcional. Disfunção vesical e intestinal em 35 crianças, a maior prevalência no sexo feminino. Conclusão: alta ocorrência de sintomas urinários e intestinais nas crianças. O sintoma mais prevalente foi constipação.
\end{abstract}

DESCRITORES: Saúde da criança. Incontinência urinária. Constipação intestinal. Estomaterapia.

\section{URINARY AND INTESTINAL SYMPTOMS IN PUBLIC ELEMENTARY SCHOOL CHILDREN}

\begin{abstract}
Objective: survey the occurrence of urinary and intestinal symptoms in children from public elementary schools in the capital of Paraná. Method: quantitative, exploratory-descriptive and cross-sectional study. Sample of parents/ guardians who completed the following instruments: Dysfunctional Voiding Scoring System; Rome IV criteria; Bristol scale. Analysis performed by IBM SPSS Statistics v.20.0. Data were collected from August to October 2019 Inclusion criteria were the child being enrolled and regularly attending elementary school and the delivery of the completed or partially completed questionnaire. Results: 458 questionnaires were delivered. Of those fully completed, $51.9 \%$ were girls, and the age was 7.7 years; $83 \%$ of the children had some urinary and/or intestinal
\end{abstract}

1. Pontifícia Universidade Católica do Paraná - Especialização em Enfermagem em Estomaterapia - Curitiba (PR), Brasil.

2. Universidade Federal do Paraná - Departamento de Cirurgia - Programa de Pós-graduação em Clinica Cirúrgica Curitiba(PR), Brasil.

3. Universidade de Brasília - Departamento de Enfermagem - Curso de graduação e pós- graduação em Enfermagem Brasília (DF), Brasil.

4. Universidade de Brasilia - Departamento de Enfermagem - Programa de Pós-graduação em Enfermagem - Brasilia (DF), Brasil. *Autora correspondente: arruda.drielle@gmail.com

Editor de Seção: Juliano Teixeira Moraes

Recebido: Out. 18, 2021 | Aceito: Dez. 16, 2021

Como citar: Silva CPC; Miranda JNR; Arruda DF; Martins G; Assis GM. Sintomas urinários e intestinais em crianças da rede pública de ensino fundamental. ESTIMA, Braz. J. Enterostomal Ther., 2021, 19: e3021. https://doi.org/10.30886/estima.v19.1140_PT 
symptoms of low severity. There was no significant difference in symptoms between genders, and with a significant increase in severity in children aged 7 years and under. Most prevalent symptoms: reduced voiding frequency, reduced defecation frequency, defecation effort, voiding urgency and containment maneuvers; 39.6\% of the children had Functional Intestinal Constipation. Bladder and bowel dysfunction in 35 children, the highest prevalence in females. Conclusion: high occurrence of urinary and intestinal symptoms in children. The most prevalent symptom was constipation.

DESCRIPTORS: Child health. Urinary incontinence. Intestinal constipation. Stomatherapy.

\section{SÍNTOMAS URINARIOS E INTESTINALES EN NIÑOS DE ESCUELAS PRIMARIAS PÚBLICAS}

\section{RESUMEN}

Objetivo: relevar la ocurrencia de síntomas urinarios e intestinales en niños de escuelas primarias públicas de la capital paranaense. Método: estudio cuantitativo, exploratorio-descriptivo y de corte transversal. Muestra de padres/ responsables que completaron los siguientes instrumentos: Dysfunctional Voiding Scoring System; criterios de Roma IV; escala de Bristol. Análisis realizado por el IBM SPSS Statistics v.20.0. Los datos fueron recopilados en el periodo de agosto a octubre de 2019. Los criterios de inclusión eran de niños matriculados y que frecuenten regularmente la escuela primaria y la entrega del cuestionario completo o parcialmente completo. Resultados: Se entregaron 458 cuestionarios. De los totalmente completos, 51,9 \% era de niñas, y la de edad fue de 7,7 años; 83 \% de los niños presentaron algún síntoma urinario y/o intestinal de baja gravedad. Sin diferencia significativa de síntomas entre sexos, y con incremento significativo de gravedad en niños de 7 años o menos. Síntomas más prevalentes: frecuencia miccional reducida, frecuencia evacuatoria reducida, esfuerzo evacuatorio, urgencia miccional y maniobras de contención; 39,6 \% de los niños presentaban Constipación Intestinal Funcional. Disfunción vesical e intestinal en 35 niños, la mayor prevalencia en el sexo femenino. Conclusión: alta ocurrencia de síntomas urinarios e intestinales en niños. El síntoma más prevalente fue el estreñimiento.

DESCRIPTORES: Salud del niño. Incontinencia urinaria. Estreñimiento intestinal. Estomaterapia.

\section{INTRODUÇÃO}

Existe forte associação entre sintomas de trato urinário inferior (STUI) e sintomas intestinais na população pediátrica. Desse modo, a International Children's Continence Society (ICCS) define um termo abrangente denominado de disfunção vesical e intestinal (DVI) como sendo um grupo de sintomas que incluem alterações vesicais associadas a sintomas de funcionamento intestinal ${ }^{1,2}$.

Dentre alguns desses sintomas, pode-se citar: aumento ou diminuição da frequência urinária (<3 micções/dia), incontinência, urgência, noctúria, esforço, hesitação, disúria, manobras de contenção e retenção. São exemplos de sintomas intestinais: incontinência, esforço, dor evacuatória e constipação funcional. $\mathrm{Na}$ ausência de sintomas intestinais, o termo disfunção de trato urinário inferior (DTUI) é mais adequado ${ }^{1,2}$.

Em termos epidemiológicos, a prevalência de STUI é relevante. Por exemplo, em um estudo realizado na Lituânia, com uma amostra de 360 crianças e 388 adolescentes atendidos no hospital infantil por problemas não relacionados à micção, verificou-se que 44,3\% dos avaliados apresentavam STUI, dentre eles 34\% apresentaram urgência, 24,6\% incontinência urinária e 8,7\% enurese 3 .

No Reino Unido, em 2017, 900.000 crianças e adolescentes conviviam com a enurese, incontinência urinária diurna, encoprese e/ou constipação. Ou seja, pelo menos dois estudantes em cada sala de aula de cada escola por todo Reino Unido apresentavam $\mathrm{DVI}^{4}$.

A cada ano na Inglaterra, 15.000 crianças e adolescentes são internados nos hospitais para serem tratados por quadros de constipação crônica e infecção do trato urinário, sendo que $80 \%$ dessas internações poderiam ser evitadas, economizando recurso daquele sistema de saúde ${ }^{4}$. 
Quanto à realidade brasileira, há poucas pesquisas que avaliam a prevalência de STUI em crianças em idade escolar. Em um estudo realizado em Pelotas, ao analisar informações de 3.602 crianças entre 6 e 7 anos, identificou-se como sintomas mais prevalentes a urgência miccional (22,7\%) e manobras de contenção (38,2\%). A prevalência de enurese noturna foi de $10,6 \%$, sendo predominante no sexo masculino 5 . Esses dados são expressivos ao considerarmos os impactos que os STUI causam na vida das crianças.

Outro estudo avaliou a percepção dos pais/responsáveis com relação à presença desses sintomas, sendo que eles acreditavam que as perdas urinárias (90\%) e demais sintomas (75\%) eram devidos ao fato da criança ser preguiçosa para ir ao banheiro, não querendo interromper as atividades de lazer, ou seja, os sintomas eram voluntários. Além disso, os responsáveis afirmavam já terem aplicado alguma medida punitiva devido à presença dos sintomas, sendo que $85 \%$ relataram já terem brigado, $35 \%$ disseram que já bateram e $50 \%$ referiram que a criança já havia sido submetida a situações constrangedoras ${ }^{6}$.

$\mathrm{Na}$ idade escolar, entre 6 e 17 anos, crianças e adolescentes são sensíveis emocional e psicologicamente à opinião dos, e afetados quando se sentem em destaque em situações constrangedoras e de insucesso. As eliminações fisiológicas, por serem culturalmente tabus e veladas, são frequentemente parte do contexto para essas situações constrangedoras. Portanto, crianças e adolescentes normalmente programam as eliminações para momentos privados, em que não sintam receios de serem expostos, em especial, aqueles que já tenham algum sintoma de $\mathrm{DVI}^{7,8}$.

O período escolar corresponde à metade das horas em que os estudantes permanecem acordados. Deste modo, espera-se que esses tenham uma média de dois a três episódios de micção durante a jornada escolar. No entanto, na escola, as necessidades e fragilidades emocionais e psicológicas muitas vezes não são respeitadas, fazendo com que a maioria dos estudantes postergue e reprima a vontade de urinar ou evacuar, tornando a escola um ambiente de risco para desenvolvimento ou piora dos STUI e de sintomas intestinais ${ }^{7,8}$.

Tendo em vista a alta prevalência de sintomas vesicais e intestinais na faixa etária escolhida, é possível perceber o pontencial que a escola e os profissionais de educação têm na observação e prevenção de complicações. Frente a isso, viu-se a necessidade de investigar a prevalência dos sintomas urinários e intestinais do cenário escolhido.

Diante do exposto, gera-se a hipótese de que crianças do ensino fundamental de escolas municipais apresentam consideráveis taxas de sintomas urinários e/ou intestinais. Deste modo, o objetivo deste trabalho foi levantar a ocorrência de tais sintomas em crianças matriculadas na rede pública de ensino fundamental em escolas de uma cidade do Paraná, Brasil.

\section{MÉTODO}

\section{Tipo de estudo}

Estudo exploratório-descritivo, de corte transversal e abordagem quantitativa, realizado em duas escolas da rede pública de ensino de uma cidade do Estado do Paraná, Brasil.

\section{População}

A população do estudo foi composta pelos pais e/ou responsáveis das crianças matriculadas no ensino fundamental de escolas da rede municipal de ensino de Curitiba.

\section{Critérios de seleção}

Após a aprovação da Secretaria Municipal de Educação, 14 escolas foram pré-selecionadas de acordo com a facilidade de acesso pelas pesquisadoras. Em seguida, foi realizado contato telefônico com cada uma delas, para apresentação e solicitação para realização da pesquisa. Duas aceitaram participar. Denominou-se E1, a escola da região norte da cidade e E2 a escola da região sul. Como contrapartida à aceitação da pesquisa, essas duas escolas receberiam, por parte das pesquisadoras, uma 
palestra aos professores, pais e interessados sobre DVI em crianças, medidas de prevenção e tratamento simples. Ambas as escolas, entretanto, não aceitaram a oferta da palestra, pela justificativa de dificuldade de tempo e de reunir as pessoas.

\section{Coleta de dados}

Para a coleta de dados foram utilizados os instrumentos Dysfunctional Voiding Scoring System (DVSS), que avalia a gravidade de sintomas urinários, critérios de Roma IV, para identificação de quadros de Constipação Intestinal Funcional, com apoio da escala de Bristol, para identificação de consistência fecal.

O DVSS é um instrumento traduzido para o português e validado transculturalmente para a realidade brasileira. Avalia a ocorrência de sintomas nos últimos 30 dias, por intermédio de uma escala Likert, variando de "nunca ou quase nunca" (0 pontos) a "quase o tempo todo" (3 pontos). Os sintomas analisados são: urgência miccional, incontinência e frequência urinária, esforço e frequência evacuatória, manobras de contenção e esforço e dor ao urinar. O DVSS também avalia o fator ambiental, considerado estressores da criança. Para esse item, a pontuação é zero quando não é identificado nenhum fator ou três quando é identificado um ou mais. Além de possibilitar a avaliação de sintomas de forma isolada, o instrumento possibilita o diagnóstico de disfunção miccional, quando crianças ou adolescentes atingem seis (sexo feminino) ou nove (sexo masculino) de escore9.

Os critérios de ROMA IV, publicados em 2016, apresentam critérios diagnósticos para Disfunções Intestinais. Foram utilizados os critérios para Constipação Intestinal Funcional - CIF (C2), que correspondem a seis perguntas de resposta "sim" ou "não", relacionadas a sinais e sintomas de constipação. Considera-se positivo o diagnóstico de CIF se duas ou mais perguntas forem assinaladas como "sim". Esse instrumento considera a ocorrência de sintomas nos últimos 30 dias e em, pelo menos, $25 \%$ das evacuações ${ }^{10}$.

A Escala de Bristol é uma representação visual da consistência fecal. As fezes podem ter consistência de 01 a 07 , sendo que quanto menor o número, mais ressecadas e quanto maior, mais amolecidas. O ideal são fezes do tipo quatro (alongada com formato de salsicha ou cobra, lisa e macia). Essa escala também foi traduzida e validada para uso no Brasi ${ }^{11}$.

Também foi elaborada uma cartilha explicativa sobre DVI na infância, para esclarecimento dessas disfunções aos pais e responsáveis. Os questionários, juntamente com o termo de consentimento livre e esclarecido, em duas vias, e a cartilha foram entregues pelas pesquisadoras às diretoras: 420 questionários para a E1 e 414 para a E2, que correspondem ao número de crianças, na faixa etária do estudo, matriculadas em cada escola. As diretoras entregaram os questionários aos professores para que esses encaminhassem aos pais via criança. Foi combinada, com as diretoras, uma data limite (após duas semanas) para devolução dos instrumentos preenchidos.

\section{Análise e tratamento dos dados}

Os questionários foram transcritos para planilha Microsoft Excel e posteriormente analisados com o programa computacional IBM SPSS Statistics v.20.0. Armonk, NY: IBM Corp. Os resultados de variáveis quantitativas foram descritos por médias, desvios padrões, medianas, valores mínimos e máximos. Variáveis categóricas foram descritas por frequências e percentuais. Para a comparação de crianças com idade até 7 anos e crianças com mais de 7 anos e, também, para a comparação de sexos, em relação ao escore DVSS, foi usado o teste não-paramétrico de Mann-Whitney. Para avaliação da associação entre o diagnóstico ROMA IV (negativo ou positivo) e idade e sexo, foi usado o teste exato de Fisher. Valores de p $<0,05$ indicaram significância estatística.

\section{Aspectos éticos}

A pesquisa foi aprovada pelo Comitê de Ética em Pesquisa da Pontifícia Universidade Católica do Paraná sob parecer $\mathrm{n}^{\circ}$ 3.575.443, do ano de 2019, respeitando todas as normas e diretrizes para pesquisas envolvendo seres humanos, apresentadas na Resolução 466/2012 do Conselho Nacional de Saúde. 


\section{RESULTADOS}

Do total de 834 questionários entregues às escolas, houve a devolução de 458 (224 da E1 e 234 da E2). No entanto, nem todos estavam completamente preenchidos. Houve falha de preenchimento em todos os instrumentos (DVSS, Critérios de ROMA IV e escala de Bristol), na idade e no sexo da criança.

Do total de questionários devolvidos, o DVSS foi preenchido em 454, os Critérios de ROMA IV em 449 e a Escala de Bristol em 446. Talvez por limitação ou falta de clareza do questionário de coleta, a maior lacuna de preenchimento foi em sexo e idade, sendo que 185 vieram preenchidos com a informação de sexo e 84 com a informação de idade. Portanto, $14,4 \%(n=66)$ vieram totalmente preenchidos. Sendo assim, diante dessa perda amostral expressiva, as autoras optaram por manter a amostra total da devolução e considerar análises isoladas.

Dos 185 questionários com preenchimento do sexo, 96 eram de meninas $(51,9 \%)$ e 89 eram de meninos $(48,1 \%)$. Quanto à idade, de 84 questionários preenchidos, a média foi de 7,7 anos, com desvio padrão de 1,6.

Do total de questionários recebidos, 184 tinham o sexo da criança e o DVSS respondidos. Desses, 16 meninos apresentaram escore maior que 9, e 38 meninas apresentaram maior que 6, fechando diagnóstico de disfunção miccional para 54 crianças $(29,3 \%)$.

Se considerarmos sintomas do DVSS isolados, 375 , ou seja, $83 \%$ das crianças $(n=454)$ apresentaram pelo menos um sintoma urinário ou intestinal. A Tabela 1 apresenta o percentual de ocorrência de cada sintoma identificado pelo instrumento, bem como a frequência de manifestação desse sintoma.

Tabela 1. Ocorrência de sintomas urinários em crianças da rede municipal de ensino fundamental de acordo com o Dysfunctional Voiding Scoring System. Curitiba (PR), Brasil - 2019.

\begin{tabular}{|c|c|c|}
\hline Pergunta & Resposta & n (\%) \\
\hline \multirow{4}{*}{1} & 0 & $410(90,3)$ \\
\hline & 1 & $33(7,3)$ \\
\hline & 2 & $6(1,3)$ \\
\hline & 3 & $5(1,1)$ \\
\hline \multirow{4}{*}{$\begin{array}{l}2 \\
\text { Quando seu(a) filho(a) se molha de xixi a cueca ou calcinha fica ensopada? }\end{array}$} & 0 & $418(92,1)$ \\
\hline & 1 & $24(5,3)$ \\
\hline & 2 & $8(1,8)$ \\
\hline & 3 & $4(0,9)$ \\
\hline \multirow{4}{*}{$\begin{array}{l}3 \\
\text { Com que frequência seu(a) filho(a) não faz cocô todos os dias? }\end{array}$} & 0 & $227(50,0)$ \\
\hline & 1 & $117(25,8)$ \\
\hline & 2 & $79(17,4)$ \\
\hline & 3 & $31(6,8)$ \\
\hline \multirow{4}{*}{$\begin{array}{l}4 \\
\text { Seu(a) filho(a) tem que fazer força para fazer cocô? }\end{array}$} & 0 & $262(57,7)$ \\
\hline & 1 & $107(23,6)$ \\
\hline & 2 & $51(11,2)$ \\
\hline & 3 & $34(7,5)$ \\
\hline \multirow{4}{*}{$\begin{array}{l}5 \\
\text { Com que frequência seu(a) filho(a) só vai ao banheiro fazer xixi uma ou duas vezes por dia? }\end{array}$} & 0 & $182(40,1)$ \\
\hline & 1 & $44(9,7)$ \\
\hline & 2 & $143(31,5)$ \\
\hline & 3 & $85(18,7)$ \\
\hline
\end{tabular}

continua... 
Tabela 1. Continuação...

\begin{tabular}{|c|c|c|}
\hline Pergunta & Resposta & n (\%) \\
\hline \multirow{4}{*}{$\begin{array}{l}6 \\
\text { Seu(a) filho(a) segura o xixi cruzando as pernas, agachando ou dançando? }\end{array}$} & 0 & $325(71,6)$ \\
\hline & 1 & $73(16,1)$ \\
\hline & 2 & $31(6,8)$ \\
\hline & 3 & $25(5,5)$ \\
\hline \multirow{4}{*}{$\begin{array}{l}7 \\
\text { Quando seu(a) filho(a) precisa fazer xixi tem que ir rápido ao banheiro? } \\
\text { (não consegue esperar) }\end{array}$} & 0 & $302(66,5)$ \\
\hline & 1 & $76(16,7)$ \\
\hline & 2 & $38(8,4)$ \\
\hline & 3 & $38(8,4)$ \\
\hline \multirow{4}{*}{$\begin{array}{l}8 \\
\text { Seu(a) filho(a) tem que fazer força para fazer xixi? }\end{array}$} & 0 & $448(98,7)$ \\
\hline & 1 & $5(1,1)$ \\
\hline & 2 & $0(0,0)$ \\
\hline & 3 & $1(0,2)$ \\
\hline \multirow{4}{*}{$\begin{array}{l}9 \\
\text { Seu(a) filho(a) disse que sente dor quando faz xixi? }\end{array}$} & 0 & $436(96,0)$ \\
\hline & 1 & $14(3,1)$ \\
\hline & 2 & $3(0,7)$ \\
\hline & 3 & $1(0,2)$ \\
\hline \multirow{2}{*}{$\begin{array}{l}10 \\
\text { Seu(a) filho(a) passou por alguma situação estressante nos últimos } 30 \text { dias? }\end{array}$} & 0 & $364(80,2)$ \\
\hline & 3 & $90(19,8)$ \\
\hline
\end{tabular}

0: nunca ou quase nunca; 1: menos que metade do tempo; 2: a metado de tempo; 3: quase todo o tempo; Exceto a questão 10, em que 0 é não e 3 é sim.

O STUI mais prevalente foi o de frequência miccional reduzida (< 3 micções/dia) (pergunta 5): dos 454 questionários com o DVSS respondido, 272 assinalaram esse sintoma (59,9\%). O segundo STUI foi o de urgência (pergunta 7) com 33,5\% de respostas positivas. O outro sintoma mais prevalente foi manobras de contenção (pergunta 6) com 28,4\%.Já os sintomas intestinais mais prevalentes foram a frequência evacuatória reduzida (pergunta 3), tendo prevalência de 50,0\%, seguido do esforço evacuatório (pergunta 4), tendo prevalência de 42,3\%. Quanto à gravidade dos sintomas, a média aritmética do escore DVSS dos 454 questionários foi de 4,7. Sendo 4,5 na E1 e 4,9 na E2.

A Tabela 2 apresenta a associação entre a gravidade de sintomas identificados pelo DVSS com a caracterização da amostra, sendo que foram 184 questionários preenchidos com DVSS e sexo, e 83 questionários com DVSS e idade. Observa-se que houve diferença estatisticamente significativa entre gravidade dos sintomas em crianças com 7 anos ou menos e as demais.

A Tabela 3 apresenta o percentual dos sintomas mais prevalentes de acordo com o sexo da criança $(n=184)$. Vale ressaltar que a mesma criança pode apresentar mais de um sintoma. Nota-se, portanto, maior ocorrência de frequência miccional reduzida (< 3 micções/dia) e de manobras de contenção nas meninas, assim como a frequencia evacuatória reduzida nos meninos chama a atenção.

Dos 449 questionários que estavam respondidos nos critérios de Roma IV, $178(39,6 \%)$ foram positivos para CIF, pois tiveram dois ou mais critérios positivos em pelo menos $25 \%$ das evacuações. Não foi identificada diferença estatisticamente significativa para CIF quando cruzada nos questionários com idade e sexo.

Analisando isoladamente os sintomas dos Critérios de Roma IV, a Tabela 4 traz a ocorrência de cada um. O sintoma mais referido foi a frequência reduzida de evacuações, seguido de fezes endurecidas e de esforço evacuatório. 
Tabela 2. Associação entre severidade dos sintomas de disfunção vesical intestinal, idade e sexo em crianças da rede pública de ensino fundamental. Curitiba (PR), Brasil - 2019.

\begin{tabular}{|c|c|c|c|c|c|c|}
\hline \multirow{2}{*}{ Idade (anos) } & \multicolumn{5}{|c|}{ Escore DVSS } & \multirow{2}{*}{$\mathrm{p}^{*}$} \\
\hline & $\mathrm{n}$ & Média & Mediana & Mínimo & Máximo & \\
\hline Até 7 & 42 & 6,7 & 6 & 0 & 20 & \multirow{2}{*}{0,015} \\
\hline Mais de 7 & 41 & 4,5 & 4 & 0 & 16 & \\
\hline \multirow{2}{*}{ Sexo } & \multicolumn{5}{|c|}{ Escore DVSS } & \multirow{2}{*}{$\mathrm{p}^{*}$} \\
\hline & $\mathrm{n}$ & Média & Mediana & Mínimo & Máximo & \\
\hline Feminino & 96 & 5,4 & 4 & 0 & 20 & \multirow{2}{*}{0,350} \\
\hline Masculino & 88 & 4,9 & 3,5 & 0 & 18 & \\
\hline
\end{tabular}

*Teste não-paramétrico de Mann-Whitney, p<0,05. DVSS = Dysfunctional Voiding Scoring System.

Tabela 3. Ocorrência dos sintomas de acordo com o sexo, segundo o Dysfunctional Voiding Scoring System mais prevalentes. Curitiba (PR), Brasil - 2019.

\begin{tabular}{lll}
\hline Sintomas urinários & Percentual & Frequência \\
\hline Meninas ( $\mathbf{n}=96)$ & & 60 \\
\hline frequência miccional reduzida & 64,6 & 32 \\
\hline urgência miccional & 33,3 & 32 \\
\hline manobras de contenção & 33,3 & 44 \\
\hline Meninos (n=88) & & 33 \\
\hline frequência miccional reduzida & 49,4 & 20 \\
\hline urgência miccional & 37,1 & 22,5 \\
\hline manobras de contenção & & 53 \\
\hline Sintomas intestinais & & 44 \\
\hline Meninas (n=96) & 55,2 & 47 \\
\hline frequência evacuatória reduzida & 45,8 & 46 \\
\hline esforço evacuatório & & \\
\hline Meninos (n=88) & 52,8 & \\
\hline frequência evacuatória reduzida & 51,7 & \\
\hline esforço evacuatório & & \\
\hline
\end{tabular}

Tabela 4. Prevalência de sintomas de constipação intestinal funcional segundo critérios de Roma IV em crianças da rede pública de ensino fundamental. Curitiba (PR), Brasil - 2019.

\begin{tabular}{|c|c|c|}
\hline Pergunta & Resposta & $\mathrm{n}(\%)$ \\
\hline 1 & não & $327(72,8)$ \\
\hline Precisa fazer força para fazer cocô? & $\operatorname{sim}$ & $122(27,2)$ \\
\hline 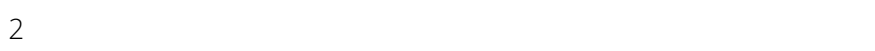 & não & $277(61,7)$ \\
\hline O cocô é seco ou duro? & $\operatorname{sim}$ & $172(38,3)$ \\
\hline 3 & não & $350(78,0)$ \\
\hline Tem a sensação de que não saiu todo o cocô? & $\operatorname{sim}$ & $99(22,0)$ \\
\hline 4 & não & $416(92,7)$ \\
\hline Tem a sensação de que tem alguma coisa que não deixa o cocô sair? & $\operatorname{sim}$ & $33(7,3)$ \\
\hline 5 & não & $445(99,1)$ \\
\hline Precisa ajudar com a mão pra tirar o cocô? & $\operatorname{sim}$ & $4(0,9)$ \\
\hline 6 & não & $268(59,7)$ \\
\hline Faz cocô três vezes na semana ou menos? & $\operatorname{sim}$ & $181(40,3)$ \\
\hline
\end{tabular}


Em relação à escala de Bristol, preenchida em 446 questionários, a Tabela 5 apresenta o percentual para cada consistência fecal. Nota-se que $71,7 \%$ das crianças têm as fezes em formatos considerados ideais (3 e 4), mas há uma tendência às fezes ressecadas em comparação com as amolecidas. Não houve diferença estatisticamente significativa na comparação de consistência fecal quando cruzada nos questionários com idade e sexo.

Tabela 5. Prevalência do formato de fezes segundo a escala de Bristol em crianças da rede pública de ensino fundamental. Curitiba (PR), Brasil - 2019.

\begin{tabular}{cc}
\hline Bristol & $\mathbf{n}(\%)$ \\
\hline 1 & $29(6,5)$ \\
\hline 2 & $82(18,4)$ \\
\hline 3 & $212(47,5)$ \\
\hline 4 & $108(24,2)$ \\
\hline 5 & $10(2,2)$ \\
\hline 6 & $3(0,7)$ \\
\hline 7 & $2(0,4)$ \\
\hline Total & $446(100,0)$
\end{tabular}

Quanto aos fatores psicossociais, dos 454 questionários respondidos no DVSS, 67 assinalaram a pergunta 10, ou seja, $14,8 \%$ das crianças foram submetidas a alguma situação estressante nos últimos 30 dias.

As situações assinaladas e/ou escritas foram: 29 apontaram para problemas em casa (sendo 14 deles morte de familiar, e 10 o divórcio), 21 pontuaram mudança de casa, 19 assinalaram problemas escolares, 18 marcaram eventos especiais (sendo 6 deles o aniversário), 14 relataram um bebê novo em casa, 10 sofream uma mudança de escola e 2 pontuaram o abuso (sendo um deles o sexual).

Considerando que a DVI é a presença de disfunção miccional somada à CIF, das 54 crianças que apresentaram disfunções miccionais, 35 eram constipadas, totalizando 35 crianças com DVI. Desse total de crianças com DVI, 23 (65,7\%) eram do sexo feminino e $12(34,3 \%)$ do sexo masculino.

\section{DISCUSSÃO}

A ocorrência de sintomas urinários ou intestinais foi de 83\%, taxa superior a estudo que identificou prevalência de $44 \%{ }^{3}$. Vale citar que as taxas foram semelhantes entre as duas escolas investigadas. Analisando a prevalência em comparação com o sexo, a maior parte dos estudos apontam uma maior prevalência de sintomas de DVI em crianças do sexo feminino ${ }^{3,6}$, porém foi encontrado um estudo no qual a prevalência foi maior em crianças do sexo masculino5. Se analisados os resultados encontrados na presente pesquisa, não houve diferença estatisticamente significativa, sendo semelhante em ambos os sexos. Porém, se considerados isoladamente os sintomas de frequência miccional reduzida e manobras de contenção, a prevalência foi maior em meninas.

Ainda considerando isoladamente os STUI, o presente estudo identificou como sintomas mais prevalentes a frequência miccional reduzida (<3 micções/dia). Esse resultado difere de outros estudos, que identificaram a urgência miccional como mais prevalente em crianças em idade escolar ${ }^{3,5,6}$. Estudos encotrados na literatura abordam o impacto que os banheiros em ambiente escolar causam nos estudantes, fazendo com que as eliminações sejam postergadas.

Vale ressaltar que o achado da baixa frequencia miccional pode estar relacionado com a baixa ingestão de líquidos duranto o período em atividades educacionais. No entanto os fatores destacados pelos estudos estão relacionados à infraestrutura, falta de privacidade para uso dos banheiros, momento ideal para eliminações, ida ao banheiro durante as aulas, sensação de insegurança e bullying ${ }^{7,8}$. Além desses pontos, a higiene do ambiente impacta os estudantes, que frequentemente se encontram com papel higiênico no chão, lixeiras lotadas e transbordando, urina no assento sanitário e no chão, odor ruim, pias sujas e molhadas, falta de papel e de sabonete ${ }^{8}$. 
Ao analisar as crianças que apresentaram DVSS condizente com disfunção miccional ( $\mathrm{n}=54$ crianças), foi identificada maior prevalência dos sintomas de urgência $(64,8 \%)$, seguida de manobras de contenção $(61,1 \%)$ e incontinência $(24,1 \%)$. Esse dado corrobora dados encontrados na literatura em estudo realizado com crianças com DTUI (amostra de 22 crianças), em que o autor identificou que os sintomas mais prevalentes foram urgeincontinência (81\%), seguidas de manobras de contenção $(77,3 \%)$ e enurese $(59,1 \%)$. Quando avaliado o sexo acometido, as disfunções estavam mais presentes no sexo feminino $(90,9 \%)^{6}$.

Ao compararmos os escores da escala DVSS para sintomas urinários e a presença de constipação intestinal (Roma IV positivo), ficou evidenciado a alta prevalência de CIF nas crianças, independente do escore de DVSS. Já em outros estudos, identificou-se associação entre altos escores no DVSS com constipação ${ }^{12-14}$.

Estudos encontrados na literatura avaliaram a associação entre maior score DVSS e fatores estressantes psicossociais apresentados pelas crianças. Essa associação foi observada em $37 \%{ }^{15}$ a $60 \%{ }^{16}$ dos casos. No presente estudo foram identificadas algumas situações consideradas estressantes para as crianças, sendo problemas em casa (divórcio dos pais e morte de familiar), problemas escolares, eventos especiais (aniversário), mudança de casa, bebê novo em casa, mudança de escola e abusos.

Além do fato de alterações e/ou estresses emocionais aumentarem a presença de STUI, é importante ressaltar também que a presença desses sintomas pode acarretar problemas emocionais e sociais. Neste trabalho não foi encontrada uma associação estatisticamente significativa entre as situações de estresse e ocorrência de sintomas urinários ou intestinais ou aumento de escore DVSS, visto que apenas 19,8\% das crianças pontuaram a questão 10 do instrumento.

Os resultados desse estudo foram condizentes com a literatura. As DVI acometem um percentual expressivo de crianças hígidas. Essas crianças ficam expostas às complicações das DVI, como dermatites associadas à umidade, esvaziamento vesical incompleto, infecção do trato urinário (ITU) e refluxo vesico-ureteral, que coloca em risco a função do trato urinário superior ${ }^{7,8}$.

Além dessas consequências físicas, a criança com DVI tem maiores chances de desenvolver alterações comportamentais e psicossociais, pois são frequentemente expostas a situações constrangedoras, que limita a vida social, diminui a autoestima e compromete o desempenho escolar ${ }^{1,2}$.

A maneira mundialmente reconhecida de prevenir a DVI e considerada a primeira linha de tratamento é a uroterapia. Ela consiste em abordagem não cirúrgica e não farmacológica que envolve a educação da criança/adolescente e seus cuidadores/família quanto ao funcionamento do trato urinário, ingestão de fluidos e fibras, hábitos urinários, intervalo entre as micções e posicionamento no vaso sanitário ${ }^{1,2}$. Além de simples, a uroterapia é efetiva na redução de sintomas e com resolução de grande parte dos casos².

Faz-se necessário uma forte disseminação de conhecimento sobre os sintomas de DVI e das medidas preventivas e de controle junto à população, em especial à comunidade escolar. Considerando que todas as crianças em idade para manifestar a DVI estão em idade escolar e devem estar matriculadas no ensino fundamental, o professor se torna um profissionalchave na escola para a identificação de um possível problema ${ }^{8}$.

Apesar da necessidade de que os enfermeiros participem desse processo de identificação de crianças com sintomas urinários e intestinais e/ou orientação aos professores, o cenário atual mostra uma certa dificuldade e resistência desses profissionais da enfermagem se inserirem no contexto escolar. No presente estudo apenas duas escolas aceitaram participar da pesquisa, mas não autorizaram a realização da palestra educativa que seria ofertada aos pais, responsáveis e professores com orientações sobre o tema. Portanto, percebe-se a importância do tema ser abordado nos diferentes ambientes em que as crianças estão inseridas, incluindo as escolas, permitindo que haja maior interação entre as áreas da educação e saúde.

Se os professores souberem identificar sintomas de DVI podem orientar medidas e até mesmo encaminhar a criança para um serviço especializado. Por outro lado, se souberem quais são as medidas que previnem tais manifestações, podem, por meio de suas orientações, reduzir a prevalência do problema no ambiente escolar ${ }^{8}$.

Segundo o Conselho Federal de Enfermagem (COFEN) e a lei do exercício profissional, uma das atribuições do enfermeiro é participar em atividades de educação em saúde com o objetivo de melhorar a saúde do indivíduo, família e coletividade ${ }^{17}$. Portanto, o enfermeiro como agente de educação em saúde surge nesse cenário com a possibilidade de capacitar os professores e serem referências para a conexão entre escola e serviço de saúde ${ }^{8}$.

Além da participação educativa e orientativa dos enfermeiros, também é de sua competência atuar frente às incontinências urinárias e intestinais, bem como participar da reeducação dos hábitos miccionais e evacuatórios ${ }^{17}$. Dentro das especialidades 
do enfermeiro, destaca-se a relevância do enfermeiro estomaterapeuta. Esse profissional atua, dentre uma das suas três áreas, com a área de incontinências. Nesse aspecto, o enfermeiro estomaterapeuta tem como objetivo avaliar pacientes com alterações urinárias e/ou intestinais, por meio de instrumentos e exame físico, bem como atuar para prevenção e tratamento dessas alterações ${ }^{18}$.

Quanto às limitações desse estudo, considera-se que o preenchimento do formulário pelos pais e responsáveis foi um fator complicador. Isso porque muitos formulários não foram preenchidos, outros incompletos e em outros, ainda, notou-se dificuldade de compreenção da pergunta (especificamente em relação ao DVSS), o que pode ter interferido na resposta. Essa barreira reduziu consideravelmente a amostra. Sugere-se, para futuras pesquisas, comparar a ocorrência dos sintomas com a relação das crianças, a escola e ao o conhecimento e atitude dos professores quanto ao assunto.

\section{CONCLUSÃO}

Constatou-se alta ocorrência de sintomas urinários e intestinais em crianças matriculadas na rede municipal de ensino fundamental em duas escolas paranaenses, pois mais de $83 \%$ da amostra apresentou, pelo menos, um sintoma urinário e/ou intestinal, independentemente da severidade desse sintoma. Percebe-se que as DVI estão presentes em crianças hígidas em idade escolar. Sendo que no presente estudo, das crianças que apresentaram sintomas miccionais, mais da metade tinham também CIF, confirmando diagnóstico para DVI.

Os sintomas urinários mais prevalentes foram frequência miccional reduzida, urgência miccional e manobras de contenção. Já os sintomas intestinais mais prevalentes foram frequência evacuatória reduzida e esforço evacuatório, condizendo com a alta ocorrência de diagnóstico de constipação intestinal funcional (critérios de Roma IV positivos).

Houve diferença estatisticamente significativa para escore do DVSS entre crianças maiores e menores de 7 anos, mas entre ambos os sexos não se observou essa diferença. Meninas, no entanto, apresentaram uma maior ocorrência de frequência miccional reduzida e manobras de contenção do que meninos.

Identificou-se que crianças em idade escolar apresentam sintomas urinários e intestinais de forma significativa, sendo esse um problema real e, na maioria das vezes, não diagnosticado e nem tratado. É necessária uma ação preventiva e corretiva frente a esse problema, sendo o enfermeiro o profissional que apresenta conhecimento técnico-científico e legal para atuar junto a essa população.

\section{CONTRIBUIÇÃO DOS AUTORES}

Conceitualização: Miranda JNR, Silva CPC, Assis GM e Martins G; Investigação: Miranda JNR e Silva CPC; Redação - Primeira versão: Miranda JNR, Silva CPC e Arruda DF; Redação - Revisão \& Edição: Miranda JNR, Silva CPC e Arruda DF; Recursos: Miranda JNR e Silva CPC; Supervisão: Assis GM e Martins G.

\section{DISPONIBILIDADE DE DADOS DE PESQUISA}

Não se aplica.

\section{FINANCIAMENTO}

Não se aplica.

\section{AGRADECIMENTO}

Não se aplica. 


\section{REFERÊNCIAS}

1. Austin PF, Bauer SB, Bower W, Chase J, Franco I, Hoebeke P et al. The standardization of terminology of lower urinary tract function in children and adolescents: Update report from the standardization committee of the International Children's Continence Society. Neurourol Urodyn. 2016;35(4):471-81. https://doi.org/10.1002/nau.22751

2. Santos J, Lopes RI, Koyle MA. Bladder and bowel dysfunction in children: An update on the diagnosis and treatment of a common, but underdiagnosed pediatric problem. Can Urol Assoc J. 2017;11(1-2Supl1):S64-72. https://doi.org/10.5489/ cuaj.4411

3. Jankauskienè A, Vaičiūnienè D, Stacevičienè I. Prevalence of lower urinary tract symptoms in Vilnius area children and adolescents. Acta Med Litu. 2014; 21(1):27-33. https://doi.org/10.6001/actamedica.v21i1.2886

4. ERIC, The Children's Bowel \& Bladder Charity [internet] Bristol: ERIC, The Children's Bowel \& Bladder Charity; [citado 2019 fev 19]. ERIC's strategic plan 2017-2020; 2017. Disponível em: https://www.eric.org.uk/Handlers/Download.ashx?IDMF=c7a02cd31374-49ea-84b7-0b99d56cf4c3

5. Mota DM, Barros AJD, Matijasevich A, Santos IS. Prevalence of enuresis and urinary symptoms at age 7 years in the 2004 birth cohort from Pelotas, Brazil. J Pediatr. 2015; 91(1):52-8. https://doi.org/10.1016/j.jped.2014.04.011

6. Veloso LA, de Mello MJG, Neto JPMR, Barbosa LNF, e Silva EJC. Qualidade de vida, nível cognitivo e desempenho escolar em crianças portadoras de distúrbio funcional do trato urinário inferior. J Bras Nefrol. 2016;38(2):234-44. http://doi. org/10.5935/0101-2800.20160033

7. Tatlow-Golden M, O'Farrelly C, Booth A, Doyle O. “Bursting” to Go and Other Experiences: Children's Views on Using the Toilet in the First School Year.J Sch Nurs. 2017;33(3):214-22. https://doi.org/10.1177/1059840516646422

8. Norling M, Stenzelius K, Ekman N, Wennick A. High School Students' Experiences in School Toilets or Restrooms. J Sch Nurs. 2016;32(3):164-71. https://doi.org/10.1177/1059840515611476

9. Calado AA, Araujo EM, Barroso U, Netto JMB, Filho MZ, Macedo A et al. Crosscultural adaptation of the dysfunctional voiding score symptom (DVSS) questionnaire for Brazilian children. Int Braz J Urol. 2010;36(4):458-63. https://doi.org/10.1590/S167755382010000400009

10. Lacy BE, Mearin F, Chang L, Chey WD, Lembo AJ, Simren M et al. Bowel Disorders. Gastroenterology. 2016;150(6):1393-407. https://doi.org/10.1053/j.gastro.2016.02.031

11. Martinez AP, Azevedo GR. Tradução, adaptação cultural e validação da bristol stool form scale para a população brasileiro. Rev Lat-Am Enfermagem. 2012;20(3):583-9. http://doi.org/10.1590/S0104-11692012000300021

12. Sampaio C, Sousa AS, Fraga LGA, Veiga ML, Netto JMB, Barroso U. Constipation and Lower Urinary Tract Dysfunction in Children and Adolescents: A Population-Based Study. Front Pediatr. 2016;4. https://doi.org/10.3389/fped.2016.00101

13. Zeevenhooven J, Koppen IJN, Benninga MA. The New Rome IV Criteria for Functional Gastrointestinal Disorders in Infants and Toddlers. Pediatr Gastroenterol Hepatol Nutr. 2017;20(1):1-13. http://doi.org/10.5223/pghn.2017.20.1.1

14. Hodges SJ. Enema Regimen in Pediatric Bladder Dysfunction. Curr Bladder Dysfunct Rep. 2017;12(3):225-8. https://doi. org/10.1007/s11884-017-0447-y

15. Wolfe-Christensen C, Guy WC, Mancini M, Kovacevic LG, Lakshmanan Y. Evidence of Need to Use Self-Report Measures of Psychosocial Functioning in Older Children and Adolescents with Voiding Dysfunction. J Urol. 2016;195(5):1570-4. https://doi. org/10.1016/j.juro.2015.11.045

16. Logan BA, Correia K, McCarthyJ, Slattery MJ. Voiding dysfunction related to adverse childhood experiences and neuropsychiatric disorders. J Pediatr Urol. 2014;10(4):634-8. https://doi.org/10.1016/j.jpurol.2014.06.012

17. Conselho Federal de Enfermagem (COFEN). Parecer $n^{\circ}$ 04/2016/CTAS/COFEN. Manifestação sobre procedimentos da área de enfermagem [Internet]. Brasília: 2016. Disponível em: http://www.cofen.gov.br/parecer-no-042016ctascofen_45837.html

18. Associação Brasileira de Estomaterapia [internet]. São Paulo: Associação Brasileira de Estomaterapia; c2016 [citado 2020 Jan 14]. Competências do enfermeiro estomaterapeuta;. Disponível em: http://sobest.provisorio.ws/textod/19 